\title{
El Uso del Conocimiento Matemático en las Comunidades de Ingenieros. Del Objeto a la Funcionalidad Matemática
}

\author{
The Use of Mathematical Knowledge within Communities of Engineers. \\ From Object to Mathematical Functionality
}

\author{
E. Johanna Mendoza-Higuera * \\ ORCID iD 0000-0002-6159-5015 \\ Francisco Cordero* \\ ORCID iD 0000-0002-7891-7498 \\ Miguel Solís $^{* * *}$ \\ ORCID iD 0000-0003-3231-7865 \\ Karla Gómez ${ }^{* * * * *}$ \\ ORCID iD 0000-0003-4079-2391
}

\begin{abstract}
Resumen
En este artículo discutimos la falta de marcos de referencia para atender la justificación funcional matemática que demandan otros dominios de conocimiento como la ingeniería. La matemática escolar habitual es problematizada, considerando su función dual como herramienta y como objeto de estudio, desde la Construcción Social de la Matemática. En este sentido, con la Teoría Socioepistemológica de la Matemática Educativa, estudiamos el uso del conocimiento matemático que emerge de las comunidades de ingenieros para conformar elementos de diálogo entre la matemática y el quehacer cotidiano de la ingeniería. Para tal fin, ofrecemos tres ejemplos que cuestionan el saber matemático escolar en escenarios de la escuela y en las prácticas profesionales del ingeniero, en los cuales se valoran los usos matemáticos: simbiosis entre predicción y simulación; modelación de la acumulación y modelación de lo óptimo.
\end{abstract}

\footnotetext{
* Maestra en Ciencias Especialidad en Matemática Educativa por el Centro de Investigación y de Estudios Avanzados del IPN (CINVESTAV - IPN). Candidata a doctora en el Centro de Investigación y de Estudios Avanzados del IPN (CINVESTAV - IPN), México. Dirección postal: Departamento de Matemática Educativa, Av. IPN, 2508, San Pedro Zacatenco, Gustavo A. Madero, Ciudad de México, México, C.P: 07360. E-mail: ejmendoza@cinvestav.mx.

** Doctor en Ciencias Especialidad Matemática Educativa por el Centro de Investigación y de Estudios Avanzados del IPN (CINVESTAV - IPN). Investigador titular del Centro de Investigación y de Estudios Avanzados del IPN (CINVESTAV - IPN). Dirección postal: Departamento de Matemática Educativa, Av. IPN, 2508, San Pedro Zacatenco, Gustavo A. Madero, Ciudad de México, México, C.P: 07360. E-mail: fcordero@cinvestav.mx.

*** Doctor en Ciencias Especialidad en Matemática Educativa por Centro de Investigación y de Estudios Avanzados del IPN (CINVESTAV - IPN). Universidad Autónoma de Chiapas (UNACH), Tuxtla Gutiérrez, Chiapas. Dirección postal: Escuela de Ingeniería, Bulevar Belisario Domínguez, Kilómetro 1081, Sin Número, Terán, Tuxtla Gutiérrez, Chiapas, México. E-mail: solise@ unach.mx.

**** Doctora en Ciencias Especialidad en Matemática Educativa por el Centro de Investigación y de Estudios Avanzados del IPN (CINVESTAV - IPN). Universidad Autónoma de Yucatán (UADY), Mérida, México. Dirección postal: Anillo Periférico Norte, Tablaje Cat. 13615, Colonia Chuburná Hidalgo Inn, Mérida, Yucatán, México. E-mail: karla.GÓMEZ@ correo.uady.mx.
} 
Palabras clave: Usos del conocimiento. Cotidiano de la ingeniería. Comunidad de Conocimiento. Socialización. Socioepistemología.

\begin{abstract}
In this article, we discuss the lack of reference frames to address the mathematical functional justification demanded by other knowledge domains such as engineering. School mathematics is problematized, considering its dual function as a tool and study object, from the Social Construction of Mathematics. In this sense, with the Socioepistemological Theory of Educational Mathematics, we study the use of mathematical knowledge that emerges from the engineering communities, to form dialogue elements between mathematics and the daily engineering tasks. For this purpose, we offer three examples that question the school mathematical knowledge in school settings and in the professional engineering practices, in which mathematical uses are valued: symbiosis between prediction and simulation; Modeling of accumulation and agronomic mathematical performance.
\end{abstract}

Keywords: Uses of knowledge. Everyday of engineering. Community of Knowledge. Socialization. Socioepistemology.

\title{
1 Introducción
}

La matemática escolar, habitualmente, no tiene un marco de referencia (MR) para poder atender la justificación funcional ${ }^{1}$ que demandan otros dominios de conocimiento. Su construcción es condición sine qua non para poder crear el diálogo entre la matemática y el cotidiano disciplinar de otros dominios de conocimiento (CORDERO 2016a, 2017). En este artículo enfocamos la atención a la ingeniería, como el otro campo disciplinar. Además, nos importa declarar, desde nuestra perspectiva teórica (Teoría Socioepistemológica), la postura epistemológica y ontológica de lo que entendemos por conocimiento en los escenarios de educación, lo cual ayudará a comprender, a los lectores, los constructos diálogo y funcional.

En primer lugar, queremos decir que el desarrollo de la educación de la matemática ha considerado, entre muchos aspectos, entender el conocimiento matemático en la escuela y fuera de la escuela (CIVIL, 2002; D'AMBROSIO, 2002), y en segundo, ha logrado reflexionar para distinguir entre matemática aplicada y pura (HIRSH; MCDUFFIE, 2016), para que la primera sea usada en la vida del ciudadano y en la fuerza de trabajo (KAISER; SRIRAMAN, 2006). Ambas orientaciones - la matemática fuera de la escuela y la aplicación de las matemáticas - coinciden en un principio: relacionar la matemática con el mundo real el cotidiano de la vida y el cotidiano de las otras disciplinas - (CORDERO 2016a, 2017).

Sin embargo, la tensión radica en el constructo relación. Una asume como conocimiento verdadero el de la escuela (o el académico) por lo cual mide la emulación de ese

\footnotetext{
${ }^{1}$ Cordero y Flores (2007, p. 10) afirman que: "La justificación funcional [...] se refiere a que los mecanismos de desarrollo del uso del conocimiento en la situación específica son funcionales como contra parte de una justificación razonada, es decir, lo que norma la justificación no es una proposición lógica sino aquello que le es de utilidad a lo humano".
} 
conocimiento en el cotidiano (donde se incluye el disciplinar), y la otra, privilegia las acciones sobre la modelación matemática, es decir, se tiene que enseñar a modelar, tal vez como el matemático lo hace (CORDERO, 2017).

Por nuestra parte, aquí nuestra postura epistemológica y ontológica, sostenemos la matemática funcional $^{2}$, lo que significa que valoramos las relaciones horizontales y recíprocas entre la matemática y el cotidiano de la vida y de las disciplinas académicas (como la ingeniería) y dejamos que ahí se construyan los conocimientos matemáticos que sucedan (CORDERO, 2016a, 2016b; MENDOZA-HIGUERA, 2017). A esta construcción le

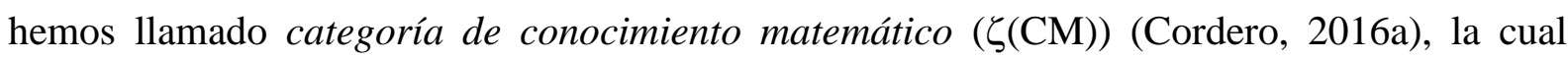
ejemplificamos en la sección de Comunidad de Conocimiento de Ingenieros.

Tal vez esa categoría alivia el hecho educativo que, en general, viven las facultades de ingeniería, en las universidades del mundo; muchas veces es el matemático o el departamento de matemáticas quien decide qué debe aprender el ingeniero, sin conocer (es la parte grave del hecho) los usos de la matemática de la comunidades de ingenieros (MENDOZA-HIGUERA, 2017).

Por esto, es necesario adentrarnos a la Construcción Social del Conocimiento Matemático (CSCM) (CORDERO et al., 2015). Los modelos educativos, en general, no han logrado esas relaciones horizontales y recíprocas. Lo que sucede en uno no sucede en el otro. En particular, si se piensa en la matemática del aula, ésta es diferente a la matemática que sucede en el cotidiano de la ingeniería ${ }^{3}$ (CORDERO, 2016a; MENDOZA-HIGUERA, 2013; NOSS; HOYLES; POZZI, 2000; BISSELL; DILLON, 2000).

Para conformar un estatus epistemológico que rinda cuentas del conocimiento matemático en esas relaciones, se requiere ubicar una dimensión social que problematice la relación de los dominios multidisciplinares. La pluralidad epistemológica tendrá que ser favorecida. Por esto, se deberá entender al conocimiento matemático como una construcción social, lo que conlleva cuestionar no en sí a la matemática, sino su función social (CORDERO, 2017). En consecuencia se deberán valorar los conceptos entorno al conocimiento, para mejorar la educación matemática: como su institucionalización, sus usos e instrumentos, sus prácticas sociales que norman sus construcciones, el cotidiano, la labor, el

\footnotetext{
${ }^{2}$ La matemática funcional se concibe como un conocimiento útil de la gente en situaciones del cotidiano de la vida y del trabajo. Este conocimiento es dinámico y su uso se resignifica dada la situación y el escenario en que emerja (CORDERO; MENA-LORCA; HUINCAHUE, 2017).

${ }^{3}$ El cotidiano de la ingeniería expresa los usos del conocimiento matemático propios de la comunidad de ingenieros. Es decir, es el conocimiento útil de una comunidad compuesto de usos y significados que se resignifican en las situaciones específicas (CORDERO, 2017; ZALDÍVAR et al., 2014).
} 
trabajo y las acciones humanas, como la identidad, entre otros (CORDERO, 2008; MENDOZA-HIGUERA; CORDERO, 2012).

Estudios con este marco darán cuenta de la función y forma del conocimiento matemático desde la condición, en nuestro caso, del ingeniero; los cuáles serán el MR para favorecer el diálogo, el cual expresará el uso del conocimiento matemático propio del ingeniero.

A todo esto, subyace la consideración del ser con otro: lo que emana elementos como organización de grupos y función de las sociedades. En ese sentido, el constructo que se formule de ingeniero debe estar cercano a comunidad con relación al conocimiento. Si hay conocimiento existe una comunidad que lo construye (MENDOZA-HIGUERA; CORDERO, 2012).

A continuación, presentamos tres ejemplos que problematizan el saber de la matemática escolar con relación a la enseñanza y aprendizaje de la matemática para la ingeniería. El estudio pone en evidencia que la matemática escolar actual no dialoga con el cotidiano de la ingeniería. Este hecho es significativo porque las categorías que reportamos emergen de los usos y significados, de los objetos matemáticos, que suceden al seno de las comunidades de los ingenieros. Estos resultados tensan y trastocan los modelos educativos dominantes y ofrecen lineamientos para formular el MR que será la base para generar el diálogo entre la matemática y la ingeniería, a través de situaciones de aprendizaje donde se problematiza la transformación del objeto matemático a la matemática funcional; o sea lo que se ha tenido a bien llamar, en la Teoría Socioepistemológica, el rediseño del discurso matemático escolar $(\mathrm{dME})^{4}$.

\section{Problemática}

Con la Teoría Socioepistemológica de la Matemática Educativa (TSME) (CANTORAL, 2013) se ha logrado formular que la matemática escolar es de naturaleza dual. Cordero $(2008,2016 a)$ plantea y justifica este hecho en el Cálculo escolar. Lara y Cordero (2007) dan evidencia de la dualidad en textos de ingeniería; Parra y Cordero (2007) centran la atención de esa dualidad en situaciones de mecánica de fluidos cuando se usa las gráficas y

\footnotetext{
${ }^{4}$ En la CSCM se reconoce la existencia de un mecanismo que norma las acciones del docente frente a la enseñanza y las del estudiante frente al aprendizaje de la matemática. Este mecanismo es un sistema de razón que provoca fenómenos como la exclusión, la opacidad y la adherencia. El Rediseño del dME es la propuesta para atacar este mecanismo y se basa, fundamentalmente, en el reconocimiento de la funcionalidad de la matemática de la gente y su incorporación al sistema educativo (CORDERO et al., 2015).
} 
las derivadas; Torres (2013) desarrolla una investigación de una comunidad de ingenieros químicos donde la dualidad se expresa en la estabilidad y en la simultaneidad de las derivadas en el análisis del funcionamiento de transformadores eléctricos; Mendoza-Higuera (2013, 2017) estudian la dualidad en el uso de la estabilidad en la matemática y en la formación de ingenieros civiles y biónicos; y Morales y Cordero (2014) diseñan una situación donde se articula el movimiento de una partícula y la simultaneidad de la primera y segunda derivada.

La dualidad es entender que, en algún momento, la matemática es el objeto de estudio y en otro momento no lo es. Por ejemplo, la matemática en el nivel universitario, por un lado es un objeto de estudio, porque efectivamente hay especialistas en formación, es decir, hay individuos que estudian matemáticas para ser matemáticos. En ese sentido, la matemática escolar trata a la matemática como un objeto de estudio.

Pero también tenemos que entender que la matemática es un instrumento para otros dominios. Existe el profesionista usuario del conocimiento matemático, no es matemático y usa la matemática, pero no como objeto de estudio. En este escenario impera la justificación funcional (CORDERO, 2016a; CORDERO; MENA-LORCA; MONTALTO, 2010; MORALES et al., 2012). Es decir, el uso del conocimiento matemático está normado por las prácticas del trabajo de un profesionista no matemático (CORDERO; MENA-LORCA; HUINCAHUE, 2017). Por ejemplo, Buendía (2011) estudia las diferencias entre la definición matemática de función periódica y lo periódico en otras profesiones; Tuyub, Cordero, y Cantoral (2009) estudian la matemática que sucede en la toxicología; Vázquez (2011) estudia el uso de las gráficas en la biología.

Otros trabajos se preocupan por la transposición didáctica de las ciencias. Por ejemplo, Cajas (2009) distingue a la ciencia y a la ingeniería como dos dominios con naturalezas de prácticas propias y no como se piensa comúnmente: la ingeniería como ciencia aplicada. En este sentido, estudia las prácticas sociales de ingeniería y cómo se trasladan a los sistemas escolares de su enseñanza. Propone una epistemología de la ingeniería que no se centre en la ciencia sino en las prácticas.

Soares (2012) distingue a la matemática que se ofrece en la enseñanza de otras disciplinas como matemáticas de servicio. Así, por medio del enfoque pedagógico y del uso de la tecnología propone el análisis de un modelo matemático para un fenómeno biológico (transmisión de la malaria). El objetivo es hacer énfasis en que las matemáticas son útiles para la biología, además de estudiar los beneficios de la tecnología para el entendimiento del modelo matemático. 
Barbosa (2009) reconoce que los modelos matemáticos son parte del discurso pedagógico de las ciencias, que figuran en las prácticas pedagógicas como argumentos para justificar conceptos o como estructuras que ordenan el estudio de los fenómenos científicos. Los modelos matemáticos son partes constitutivas del discurso pedagógico de las ciencias. Propone romper con la idea de que los modelos matemáticos son un retrato de la realidad y que los estudiantes puedan discutir la pertinencia del modelo como representación. Así, el modelo podría tomar el papel de justificar proposiciones, establecer conceptos o estructurar fenómenos.

La Matemática Educativa ha avanzado, conformando marcos teóricos que dan respuesta, no sólo a cómo se trabaja la matemática en una actividad matemática, sino también en una actividad de trabajo no matemático, sin soslayar la justificación funcional.

Se puede decir que la TSME es un modelo teórico-empírico que articula ambos aspectos, formula epistemologías de la construcción de conocimiento matemático a través de una justificación razonada y de una justificación funcional, donde la noción de práctica social esclarece esa dualidad y en consecuencia amplía considerablemente la problemática que conlleva la matemática escolar (CORDERO; FLORES, 2007; CORDERO, 2016a).

Esta visión pone en el escenario de la Matemática Educativa el rol de la justificación funcional, la cual presume de interactuar, de manera natural, con las realidades que construye el ingeniero. Podríamos decir, entonces, que la justificación funcional provee del entendimiento de cómo un sujeto construye conocimiento, pero en su condición de un sujeto situado, un sujeto que pertenece a una cultura, a una comunidad. En síntesis, nos referimos a un sujeto social cuyas vivencias le han proporcionado conocimiento. A este sujeto lo reconceptualizamos como gente que va a la escuela (o fue), trabaja y vive en una ciudad (CORDERO, 2016a; CORDERO et al., 2015).

En ese sentido, nos centramos en identificar la matemática funcional que tiene presencia durante el actuar de la gente, y así obtener una caracterización de la misma. Por esto, consideramos tres escenarios diferentes de la matemática: el conocimiento matemático, el conocimiento escolar y el conocimiento cotidiano. Cada uno de estos se encuentran interrelacionados, pues de alguna manera, las condiciones del conocimiento cotidiano, algunas veces, han dado pie al surgimiento de los conocimientos disciplinares, y entre ellos la matemática misma (CORDERO; MENA-LORCA; HUINCAHUE, 2017). Por otra parte, la matemática escolar ha sido producto de una reorganización del saber matemático, con el interés de formar ciudadanos con los conocimientos útiles que les permitan llevar a cabo sus actividades personales y sociales (CORDERO et al., 2015). 
Las relaciones entre los escenarios conllevan cuestionar varias trayectorias que atañen a la problemática. Una de ellas, el conocimiento escolar (los cursos de matemáticas) para el conocimiento de los ingenieros en formación, pareciera estar formulada en tanto que el sistema educativo busca en su programa que la matemática escolar afecte al cotidiano de la formación del ingeniero (MENDOZA-HIGUERA, 2013, 2017). Pero ¿qué se sabe del ingeniero? ¿Cómo conoce y usa el conocimiento desde su condición de ingeniero? Es decir, ¿Cómo es el uso del conocimiento matemático propio de la ingeniería?

Precisamente, este último cuestionamiento podría ser un MR que contribuya con el rediseño del $d M E$. Esto es, por el planteamiento de estas preguntas surge la necesidad de desarrollar estudios que den cuenta de la función y forma del conocimiento matemático desde la condición de las comunidades, en nuestro caso de la ingeniería, que exprese el uso del conocimiento matemático propio de esas comunidades.

\section{Comunidad de conocimiento de ingenieros}

Todo ingeniero pertenece al menos a una comunidad de conocimiento, según sea su especialidad u oficio, su ámbito laboral o institucional. Un ingeniero es considerado como aquel que dada sus actividades cotidianas, de la ingeniería, se encuentra en interacción con otras comunidades de conocimiento. Se encuentra presente en diversas situaciones. Considerando a una situación como toda acción del ingeniero que forma parte de su vida diaria, en el seno de la ingeniería. Sin embargo, no todas las situaciones nos van a interesar. La atención se centrará en aquellas en donde se hace un uso de conocimiento matemático (CORDERO et al., 2015; MENDOZA-HIGUERA, 2013, 2017).

Entonces, a partir de una situación $\left(S_{i}\right)$, en el cotidiano de la ingeniería, sucede una comunidad de conocimiento matemático del ingeniero $\left(\mathrm{CCM}\left(\mathrm{I}_{\mathrm{i}}\right)\right)$. Es en estas situaciones en donde interactúan las comunidades de conocimiento. Todo lo anterior en su conjunto conforma el cotidiano del ingeniero (MENDOZA-HIGUERA, 2013, 2017). La naturaleza de la $S_{i}$ que definirá la $\mathrm{CCMI}_{\mathrm{i}}$ corresponderá a una categoría de modelación ${ }^{5}$ (CORDERO, 2011, 2016a; CORDERO; MENA-LORCA; HUINCAHUE, 2017). Misma que aportará elementos para caracterizar la matemática funcional de la ingeniería.

\footnotetext{
${ }^{5}$ En la TSME, la modelación es una práctica plasmada como la argumentación de la situación en cuestión, la cual está compuesta de significaciones o resignificaciones con sus respectivos procedimientos: se van construyendo de acuerdo con las operaciones que los participantes son capaces de hacer, con las condiciones que ellos son capaces de capturar y transformar y con los conceptos que van construyendo (CORDERO, 2016a).
} 
Las $S_{i}$ del ingeniero civil se basa en prácticas de diseño, construcción o ejecución, y control o conservación de estructuras, infraestructuras de transporte y obras hidráulicas (UNACH, sf); y las $S_{i}$ del ingeniero agrónomo se basa en describir, analizar y evaluar los sistemas de recursos naturales, producción vegetal y producción animal para resolver problemas prácticos del ámbito rural (GÓMEZ, 2015).

En ese sentido, a continuación presentamos tres ejemplos de usos del conocimiento matemático de la ingeniería: simbiosis entre predicción y simulación; modelación de acumulación y modelación de lo óptimo. Estas tres categorías emergieron en las comunidades de ingenieros, en cuestión: son los usos y significados, de esas comunidades, que otorgan a los objetos matemáticos: estabilidad de la ecuaciones diferenciales lineales con coeficientes constantes y optimización, respectivamente.

\subsection{Simbiosis predicción-simulación. El comportamiento tendencial de las funciones y la estabilidad de las ecuaciones diferenciales como un proceso del RdME}

En esta sección se formula el estatus epistemológico del comportamiento tendencial de las funciones (ctf) en la matemática escolar y su conexión con la estabilidad de las ecuaciones diferenciales como diálogo entre la matemática y la ingeniería, donde la relación recíproca y horizontal transforma al objeto matemático en una matemática funcional, donde la simbiosis predicción y simulación es la resignificación de usos.

Hay diferentes maneras, en la matemática escolar habitual, de explicar lo que es una ecuación diferencial. En los libros de texto de ecuaciones diferenciales usados tradicionalmente en facultades de ingeniería en México, se encuentra que: se le da sentido a la ecuación diferencial haciendo un tratamiento análogo a las ecuaciones algebraicas; se identifican situaciones que le den sentido a la ecuación diferencial y se precisa sobre la naturaleza de su solución; se describen características de la ecuación diferencial a partir de su aspecto utilitario, como una operación que resuelve problemas y se admite una descripción formal, como una relación entre la variable, la función y la derivada (EDWARDS; PENNEY, 1993; BOYCE; DIPRIMA, 1992; ZILL, 1997).

Ante la diversidad, se privilegia el contexto algebraico debido a que desarrollan métodos cuantitativos para encontrar soluciones a las ecuaciones diferenciales. Con estos métodos se podrían encontrar soluciones explícitas como fórmulas o series infinitas (CORDERO et al., 2016). Por ejemplo, si la ecuación diferencial es $y^{\prime}+y=x$, la solución es 
$y=x \quad 1+B e^{x}$, considerando el método llamado factor de integración y si la ecuación diferencial es $y^{\prime \prime}+y^{\prime}+y=x$, la solución es $y=x+1+e^{1 / 2 x}\left(C_{1} \cos \sqrt{3} / 2 x+C_{2} \operatorname{sen} \sqrt{3} / 2 x\right)$, considerando el método, por un lado, hallar la solución característica $y_{c}$ determinada por el polinomio característico $P_{2}(r)$ de la ecuación diferencial homogénea y, por el otro, hallar la solución particular $\left(y_{p}\right)$ a través del método variación de parámetros. La solución general esta compuesta por la suma de las soluciones anteriores: $y=y_{c}+y_{p}$.

Sin embargo, con estos métodos, que permanecen en la matemática escolar habitual, poco podríamos decir de las ecuaciones $y^{\prime}+y=x, \mathrm{y}, y^{\prime \prime}+y^{\prime}+y=x$, como modelos que reproducen comportamientos. Por ejemplo, la primera expresión sería un modelo donde $y$ reproduce un comportamiento similar a $x$; y la segunda expresión sería un modelo donde $y$ reproduce un comportamiento similar a $x$. El escenario cambia, ahora el foco de atención no está en resolver la ecuación, sino en establecer comportamientos con tendencia (CORDERO et al., 2016). El aspecto importante de este hecho radica en que emerge en las comunidades de ingenieros cuando se involucran en situaciones de sistemas de control (MENDOZA-HIGUERA, 2017).

Este hecho provoca cuestionamientos, en la matemática escolar habitual para ingenieros, de las ecuaciones diferenciales como $a_{n} y^{(n)}+a_{n 1} y^{(n 1)}+\ldots+a_{2} y^{\prime \prime}+a_{1} y^{\prime}+a_{0} y=f$. Por ejemplo, ¿Cuáles son las condiciones que deben cumplir esas ecuaciones diferenciales para que la solución y tienda a $f$ ? En general, ese cuestionamiento no se incluye en esos cursos; sin embargo, para $n=2$, se precisan las condiciones de $a_{0}, a_{1}$ y $a_{2}$ para que $y$ tienda en forma y rapidez al comportamiento de $f$, en ámbitos de la ingeniería (CORDERO; SOLÍS, 1997; SOLÍS, 2012, CORDERO; DUBINSKY; FERRARI, 2011, CORDERO et al., 2016).

La variación de los coeficientes juega un papel fundamental y hace concebir a la función como una instrucción que organiza comportamientos (CORDERO et al., 2016). La expresión $y^{\prime}+y=f$ representa una situación en la que, dado el comportamiento de $f$, la función $y$ tiende a comportarse como $f$. Así, la ecuación diferencial expresada como $a y^{\prime}=f \quad y$ significa que $a$ determina la rapidez con que $y$ tiende al comportamiento de $f$ en la situación analizada. 
En este sentido, el punto significativo no radica en hallar la solución, sino más bien en determinar comportamientos tendenciales entre la ecuación diferencial y su solución, y con ello lograr simulaciones. Un ejemplo de este tipo de procesos sería encontrar el coeficiente a de la ecuación $a y^{\prime}=f \quad y$ para que $y$ tienda rápidamente o lentamente hacia $f$ (CORDERO, 1998). La inmersión con comunidades de ingenieros, en diferentes experiencias de investigación, nos permiten sostener la creencia de lograr el diálogo recíproco entre la matemática escolar y el cotidiano de la ingeniería; la horizontalidad consiste en que la ecuación diferencial y la reproducción de comportamientos tienen el mismo valor epistemológico (MENDOZA-HIGUERA, 2017).

\subsubsection{La simbiosis entre la predicción y la simulación. Un diálogo entre la matemática escolar y el cotidiano de la ingeniería}

Buscar comportamientos tendenciales en las ecuaciones diferenciales lineales es producto de la simbiosis predicción-simulación (SOLÍS, 2002). Esto es, las ecuaciones diferenciales lineales con coeficiente constante (el objeto matemático) son modelos que organizan comportamientos entre dos funciones ( $f$ y $y$ ) (un significado que emerge en la ingeniería); la simbiosis predicción-simulación es la situación de tal organización puesto que para anticipar el comportamiento de $y$ se requiere de la variación de los parámetros de la ecuación, $a y^{\prime}=f \quad y$ (usos del objeto matemático que emerge en la ingeniería) (CORDERO et al., 2016; MENDOZA-HIGUERA, 2013). Con la simbiosis, como un eje, hemos generado cursos de ecuaciones diferenciales en diversas facultades de ingeniería, donde se reconocen patrones de comportamiento de $y$. Los momentos de la situación son los siguientes (SOLÍS, 2012; CORDERO et al., 2016; MENDOZA-HIGUERA, 2013).

Veamos el Cuadro 1:

\begin{tabular}{|l|l|l|}
\hline 1. Si $\begin{array}{c}a y^{\prime}+y=f \\
y^{\prime}+a y=f \\
y^{\prime}+y=a f\end{array}$ & $\rightarrow$ & ¿Cómo es el comportamiento de la \\
solución $y ?$
\end{tabular}




$$
\begin{array}{|l|l|l|}
y=A f(a x+b)+B & & \\
\hline
\end{array}
$$

Cuadro 1 - Secuencia de patrones de comportamiento

Fuente: SOLÍS (2012)

El estatus epistemológico del comportamiento tendencial, en las ecuaciones diferenciales, en la matemática escolar advierte del privilegio de los métodos cuantitativos para resolver dichas ecuaciones. Este hecho trae, inevitablemente, consecuencias didácticas (SOLÍS, 2002). Nuestras investigaciones tratan de dar cuenta de lo que significa tal comportamiento tendencial, y de sus procedimientos, en ámbitos de la enseñanza y aprendizaje de la matemática en el nivel universitario (CORDERO; RODRÍGUEZ; SOLÍS, 2013).

Éstos se confrontan con los privilegios de la enseñanza de las ecuaciones diferenciales, los cuales han provocado que los estudiantes de ingeniería no logren resignificar ${ }^{6}$ (predecir y simular) las ecuaciones diferenciales. Tales privilegios opacan procedimientos para identificar patrones gráficos y analíticos (CORDERO et al., 2015).

La categoría comportamiento tendencial de las funciones $(\zeta$ (ctf)) dialoga con el cotidiano de la ingeniería y con la matemática escolar, en este caso las ecuaciones diferenciales lineales con coeficientes constantes. En varios estudios, en diferentes años, cerca de dos décadas, se han hecho inmersiones con comunidades de conocimiento matemático de ingenieros, donde la simbiosis predicción-simulación ha sido el entorno de esas comunidades donde sucede el diálogo recíproco entre la matemática escolar y el cotidiano del ingeniero (SOLÍS, 2012, 2002).

En algún sentido, la categoría es un proceso permanente que acompaña a la funcionalidad matemática de los ingenieros y de los que están en formación. Por ejemplo, en el dominio de la ingeniería civil, el estado sólido es problematizado por el estado permanente; suceden patrones de comportamientos con tendencia de cierto tiempo en adelante. Estos patrones son los instrumentos para predecir y simular los fenómenos del estado sólido.

Pero también, la categoría permanece en situaciones de calor, cuando un instrumento es calentado y pasa de un estado a otro: los niños (educación básica) en el entorno de la simbiosis predicción-simulación generan comportamientos con tendencia al sentir la temperatura de algo que se calienta y enfría (SOLÍS, 2002, 1999). Además, en estudiantes de ingeniería con dificultades de aprender los métodos cualitativos de las ecuaciones

\footnotetext{
${ }^{6}$ La resignificación expresa la movilidad de los usos y significados del conocimiento matemático en las diferentes situaciones específicas, propias de otros dominios de conocimiento y del cotidiano de la vida.
} 
diferenciales, logran generar y revelar comportamientos con tendencia con el entorno de la simbiosis de esas ecuaciones (SOLÍS, 2002, 1999).

La categoría $\zeta$ (ctf) permanece en la matemática funcional de la gente que va a la escuela, que tiene una profesión y un cotidiano mundano (CORDERO; MENA-LORCA; HUINCAHUE, 2017).

\subsection{Modelación de la acumulación. Una comunidad de conocimiento de ingeniería civil en un escenario de la escuela}

La enseñanza y el aprendizaje de las matemáticas en la ingeniería conllevan una problemática amplia y compleja. En esencia, aquí problematizamos dos aspectos centrales. Por un lado, en la enseñanza de la matemática para la ingeniería, en general, prevalece el dominio de la matemática por encima del conocimiento de la ingeniería (MENDOZAHIGUERA, 2013, 2017; CORDERO; RODRÍGUEZ; SOLÍS, 2013). Esto conlleva formular enseñanzas de la matemática ignorando los usos del conocimiento matemático en la propia ingeniería (MENDOZA-HIGUERA, 2013). Por otro lado, como ya se mencionó, la matemática escolar habitual no tiene un marco de referencia que ayude a resignificar el conocimiento matemático para transitar en otros dominios de conocimiento, como la ingeniería (CORDERO, 2016a, MENDOZA-HIGUERA, 2013; 2017). Esto conlleva construirlo, tomando como base una epistemología de la matemática desde el ámbito de la ingeniería, donde el objeto de estudio no es en sí la matemática, sino un objeto propio de la ingeniería (por ejemplo la estabilidad) donde el conocimiento matemático es funcional y situacional (MENDOZA-HIGUERA, 2013, 2017; CORDERO et al., 2015, 2016).

Para abordar tal problemática, es obligado constituir programas de investigación para identificar los usos del conocimiento matemático (U CM) en el cotidiano disciplinar de la ingeniería, pero también importa resaltar la dimensión social, la cual en la Teoría Socioepistemológica consiste en conceptualizar a los participantes como ciudadanos miembros de una comunidad de conocimiento, donde elementos de reciprocidad, intimidad y localidad caracterizan lo propio de esta comunidad (CORDERO, 2016a; MENDOZAHIGUERA, 2013, 2017).

Con el marco anterior, presentamos, a continuación, un estudio de usos de la graficación, en una situación de acumulación de fluidos propia de las prácticas de una comunidad de conocimiento matemático de ingenieros civiles en formación $\left(\mathrm{CCM}\left(\mathrm{IC}_{\mathrm{F}}\right)\right)$. $L a$ situación significa a la ecuación diferencial lineal como un modelo de estabilidad. Donde la 
categoría del comportamiento tendencial de las funciones emerge como una argumentación propia de las participantes (MENDOZA-HIGUERA, 2013).

\subsubsection{Comunidad de conocimiento matemático de ingenieros civiles}

En las ingenierías, suceden comunidades de conocimiento en escenarios académicos y de trabajo. En el primero, interactúan ingenieros en formación, maestros, ingenieros docentes construyendo una jerga común y compartiendo situaciones propias de su disciplina. En el segundo, en el ámbito del trabajo suceden prácticas específicas como: diseñar, ejecutar y controlar (CAJAS, 2009).

El método ingenieril, se fundamenta en el aprendizaje y en el pensamiento creativo, el cual se basa en la detección de necesidades, en el diseño de productos, en la implantación del diseño y en la evaluación de resultados. Sin embargo, la esencia del método ingenieril, en términos generales, es el diseño (VALENCIA, 2004 citado en TORRES 2013).

Ahí, en el diseño, se observan procesos de análisis, toma de datos, identificación de sistemas de conocimiento y construcción de unos modelos de la realidad. No obstante, esas prácticas son soslayadas en los cursos de matemáticas en la formación inicial de ingenieros, debido a que, en general, los métodos matemáticos son enseñados sin poner en juego las situaciones de donde emergieron (MENDOZA-HIGUERA, 2013).

Prácticas como las mencionadas anteriormente, suceden en la ingeniería civil y surgen en diversas situaciones específicas. Por ejemplo, el funcionamiento hidráulico de una presa, donde se acumulan los volúmenes recibidos por un cauce y que se controlan por una cortina según la capacidad deseada. Si el volumen acumulado sobrepasa el nivel máximo, estos serán desalojados por otra estructura diseñada para tal fin (MENDOZA-HIGUERA, 2013).

En ese ámbito académico se conformó una comunidad de ingenieros en formación para reconocer sus usos del conocimiento matemático. Los ingenieros en formación participantes cursaban el sexto semestre de la Licenciatura en Ingeniería Civil en la Universidad Autónoma de Chiapas. Ya habían cursado asignaturas como Mecánica de materiales, Mecánica de suelos, Materiales de construcción y Topografía. En las sesiones de entrevista, participaron tres mujeres y un hombre.

\subsubsection{Situación específica y epistemología. La acumulación de fluidos}


Seleccionamos una situación específica, la cual le hemos llamado: acumulación de un fluido. Esta presume estar en el ámbito de la ingeniería: por un lado, aparece en la ingeniería en formación y, por el otro lado, aparece en la jerga disciplinar de la ingeniería (SOLÍS, 2012; MENDOZA-HIGUERA; CORDERO, 2012).

La situación hace referencia a la necesidad de mantener el nivel de un fluido (el movimiento del fluido es suave, lineal y sin perturbaciones) acumulado en un tanque cilíndrico al que le entra un fluido con un gasto constante y sale a través de una válvula con un gasto variable. Se considera la resistencia $R$ para el flujo del líquido en el tubo de salida, la cual se define como la diferencia del nivel del líquido necesaria para producir un cambio en una unidad en la velocidad del flujo.

La última expresión en la Figura 1 modela la dinámica del nivel del líquido en un recipiente cilíndrico.
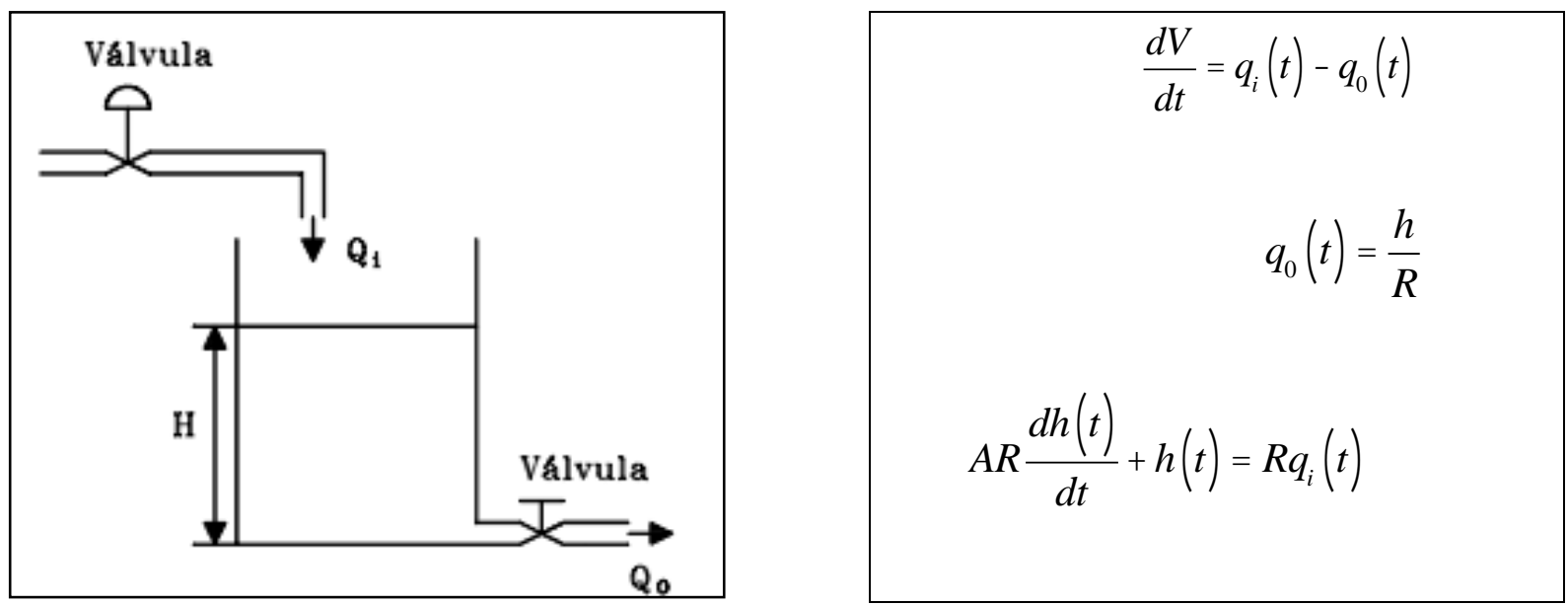

Figura 1 - Sistema de nivel de líquidos Fuente: MENDOZA-HIGUERA (2013).

La problemática principal de la situación es controlar el nivel del líquido dentro del tanque. Para ello, primero se debe entender su dinámica. Así, $h(t)$ tiende asintóticamente (de manera creciente o decreciente) a un valor constante, en este caso a $R q_{i}(t)$. Es decir, la ecuación diferencial modela un fenómeno estable. En el análisis de la dinámica del sistema, surge la búsqueda de la tendencia y así la argumentación de la estabilidad, a través de la categoría Comportamiento Tendencial de las Funciones $(\zeta$ (ctf)). la cual origina usos de las gráficas y la alternancia de dominios entre lo analítico y lo gráfico (MENDOZA-HIGUERA, 2013).

La gráfica de los comportamientos de las variables con tendencia son significados desde el mismo dominio de la ingeniería, conlleva procedimientos analíticos y de graficación; 
dentro de los primeros, emergen restas y desigualdades y de los segundos, surgen procedimientos como variar parámetros, realizar ajustes, múltiples realizaciones, construir patrones, éstos apoyados, en alguna medida, por el uso de la tecnología (MENDOZAHIGUERA, 2013) (Cuadro 2).

\begin{tabular}{|c|c|}
\hline & Situación de Transformación \\
\hline $\begin{array}{l}\text { Significac } \\
\text { iones }\end{array}$ & - $\quad$ Tendencia y comportamiento \\
\hline $\begin{array}{l}\text { Procedim } \\
\text { ientos }\end{array}$ & $\begin{array}{ll}\text { - } & \text { Buscar patrones de } \\
\text { comportamiento } \\
\text { - } & \text { Buscar la tendencia de las } \\
\text { variables cuando } \mathrm{I} \text { crece } \\
\text { - } \quad \text { Graficar y realizar ajustes para } \\
\text { encontrar un patrón deseado } \\
\text { - } \quad \text { Variar parámetros de los } \\
\text { coeficientes }\end{array}$ \\
\hline $\begin{array}{l}\text { Instrumen } \\
\quad \text { tos }\end{array}$ & $\begin{array}{l}\text { La función como una instrucción } \\
\text { que organiza comportamientos }\end{array}$ \\
\hline $\begin{array}{l}\text { Argument } \\
\text { ación }\end{array}$ & $\begin{array}{l}\text { Comportamiento tendencial de las } \\
\text { funciones }\end{array}$ \\
\hline
\end{tabular}

\begin{tabular}{|ll|}
\hline \multicolumn{1}{|c|}{ Situación de Acumulación } \\
\hline- & Acumulación \\
- & Nivel del líquido \\
- & Equilibrio de salida y entrada \\
\hline- & $\begin{array}{l}\text { Comparar el } \\
\text { comportamiento del gasto } \\
\text { de entrada y el de salida. } \\
\text { Buscar la tendencia en el } \\
\text { comportamiento cuando r } \\
\text { crece }\end{array}$ \\
\hline $\begin{array}{l}\text { La función como instrucción que } \\
\text { organiza comportamientos estables }\end{array}$ \\
\hline $\begin{array}{l}\text { Comportamiento tendencial de las } \\
\text { funciones }\end{array}$ \\
\hline
\end{tabular}

Cuadro 2 - Epistemología de uso de gráficas en una situación de acumulación de fluidos Fuente: MENDOZA-HIGUERA (2013, p. 34).

En el Cuadro 2 se presentan los aspectos esenciales de la situación específica de acumulación de un fluido: los significados, los procedimientos, los instrumentos y las argumentaciones. Para ello, se consideró la situación de transformación (CORDERO, 2008) como base para la epistemología de la situación de acumulación (MENDOZA-HIGUERA, 2013). Significar la acumulación, el nivel del líquido, los gastos de salida y entrada como variables con un comportamiento, concede procedimientos como comparar y buscar la tendencia entre los comportamientos. Es así, como las funciones que conforman la ecuación diferencial se convierten en instrucciones que organizan estos comportamientos. Estos aspectos son procesos que conllevan la construcción propia de la comunidad de conocimiento matemático de los ingenieros

\subsubsection{Los usos de la graficación en la significación de la estabilidad. Un aprendizaje de la matemática}

El diseño de situación de aprendizaje se elaboró con base en la epistemología mencionada anteriormente (Cuadro 2). El diseño estuvo conformado por tres momentos: 1. 
Significaciones de acumulación, nivel del líquido, fluido y equilibrio. 2. Construcción de modelos analíticos y gráficos. Una alternancia entre los dos dominios 3. El uso de la gráfica en la argumentación (MENDOZA-HIGUERA, 2013).

En la situación de aprendizaje se buscó que los ingenieros en formación explicaran la relación existente entre el gasto de salida (variable que depende del nivel del fluido) y el nivel del fluido en el tanque. Inicialmente, se plantearon los siguientes cuestionamientos: se tiene un tanque o depósito cilíndrico con dimensiones conocidas que contiene cierto fluido. A éste entra, el mismo fluido, con un gasto de entrada constante y sale con un gasto variable a través de una válvula ubicada en la parte inferior del tanque con diámetro conocido. ¿Cómo se calcula lo que se acumula del fluido en cualquier tiempo t? Y se acompañó la discusión con las siguientes preguntas: ¿De qué depende que haya acumulación? ¿Qué pasa con el nivel líquido cuando hay o no acumulación? ¿Cómo se comporta el gasto de salida? (MENDOZA-HIGUERA, 2013).

La CCM ( $\left.\mathrm{IC}_{\mathrm{F}}\right)$ significó la estabilidad como el equilibrio, entendido como la igualdad entre el gasto de entrada y el de salida (Ver Cuadro 3). Así mismo, emergieron procedimientos como el ajuste de patrones de tendencia de la variación del nivel del agua a través de justificaciones funcionales como graficar la estabilidad, buscando ajustar la curva a un valor constante a través del tiempo (donde el gasto de entrada es igual al gasto de salida).

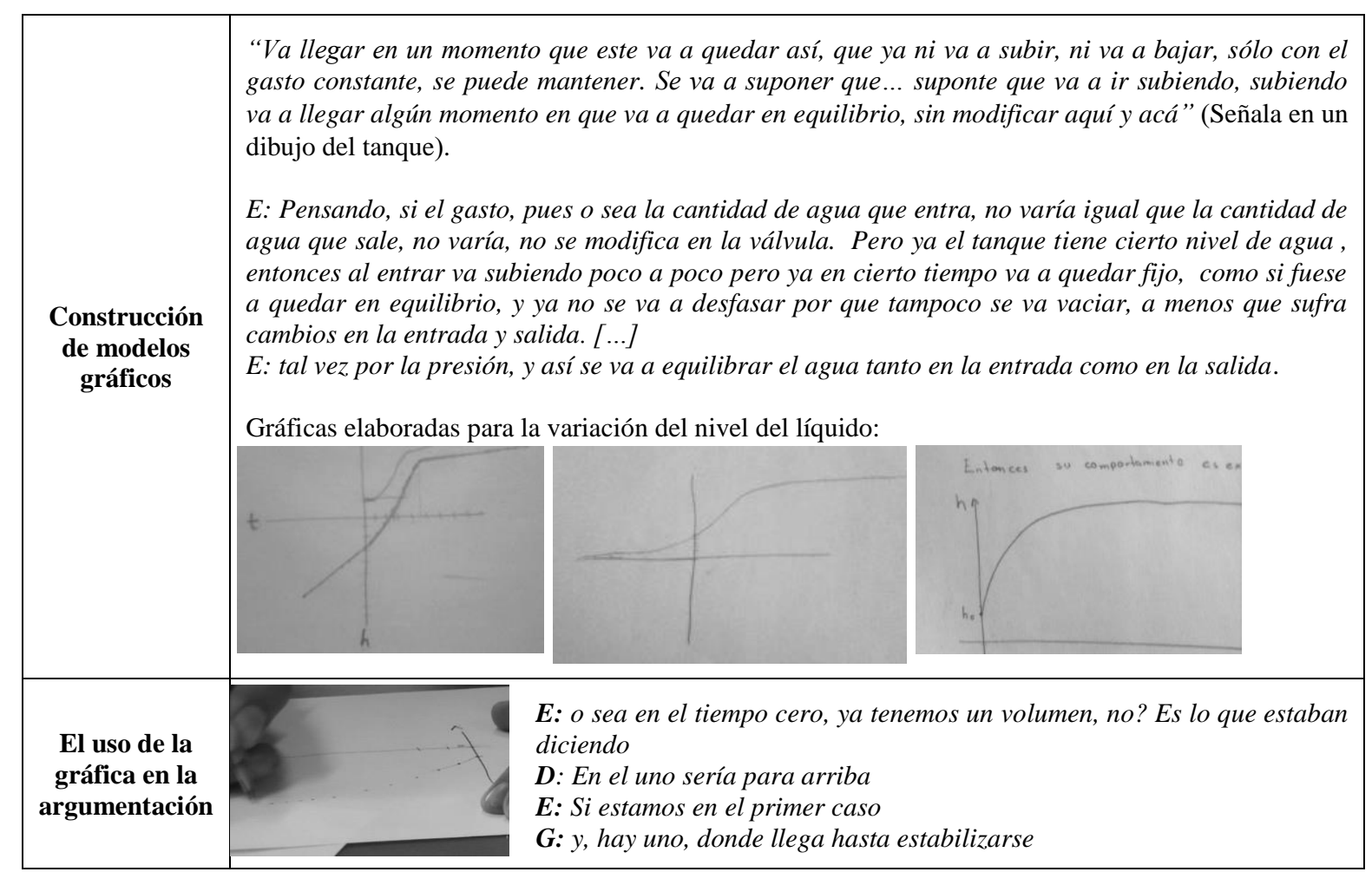

Cuadro 3 - Momentos y evidencias Fuente: MENDOZA-HIGUERA (2013). 
Con base en los usos de la gráfica, se identificaron modelos de comportamientos tendenciales desde lo gráfico y lo analítico. Los procedimientos e instrumentos que dieron forma a estos modelos gráficos y analíticos fueron: variación de parámetros, simulaciones en la calculadora hasta obtener un patrón deseado, realizaciones de ajustes tanto analíticos como gráficos, comparaciones entre los comportamientos de las variables y sus variaciones, todo estos procedimientos conllevaron la instrucción que organiza comportamientos.

En cuanto a los funcionamientos y formas del uso de las gráficas ${ }^{7}$ se evidenciaron los siguientes:

- La distribución discreta de puntos como herramienta para dibujar una curva con tendencia, al analizar las condiciones del nivel líquido y el gasto de salida y mantener el argumento del cft.

- El análisis de la curva en tanto a su concavidad para decidir cual modelaba el comportamiento tendencial. Este análisis se hizo desde la misma situación al sostener el momento de equilibrio que se debía alcanzar (MENDOZA-HIGUERA, 2013).

Al caracterizar lo propio de la comunidad de conocimiento matemático a la cual pertenecen los ingenieros en formación, hacemos uso del Modelo de Comunidad de Conocimiento Matemático. Así, elementos de Reciprocidad e Intimidad revelan lo propio del conocimiento desde el cotidiano del ingeniero y los ejes de Institucionalización e Identidad, como referentes para apreciar la continuidad del conocimiento y legitimar el nuevo conocimiento (CORDERO, 2016a).

Así, revelamos una intimidad en la construcción de conocimiento cuando generan un argumento de estabilidad en el análisis de los patrones de tendencia con la variación, en la situación específica. A continuación, se dibujan algunos elementos identificados en esta $\left(\mathrm{CCM}\left(\mathrm{IC}_{\mathrm{F}}\right)\right)($ Ver Figura 2$)$.

\footnotetext{
${ }^{7}$ El funcionamiento se manifiestan por las tareas que componen la situación, y la forma del uso será la clase de esas tareas. Las tareas pueden ser actividades, acciones, ejecuciones y alternancias de dominios. Cuando la alternancia de tareas sucede, se genera una nueva función orgánica, que debatirá con las formas de los usos. A este acto de uso se le llamará resignificación de usos (CORDERO; FLORES, 2007).
} 


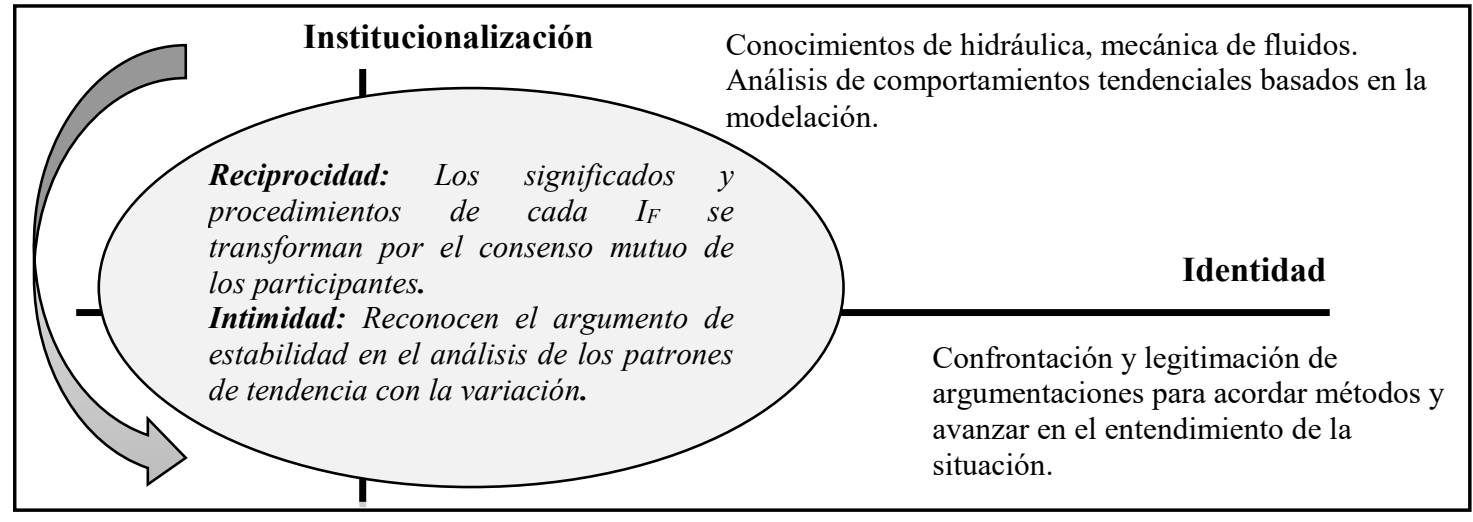

Figura 2 - Elementos de la $\mathrm{CCMI}_{\mathrm{F}}$

Fuente: MENDOZA-HIGUERA (2013).

\subsection{Modelación de lo óptimo. El conocimiento matemático de una comunidad de conocimiento de ingenieros agrónomos}

La problemática, como ya fue señalada, radica en la no consideración del uso del conocimiento matemático, en la matemática escolar; en nuestro caso, en la formación de los ingenieros agrónomos (CORDERO et al., 2015). Lo que pasa en la matemática escolar de los ingenieros en formación es independiente del cotidiano del ingeniero agrónomo. Pareciera que se le exige, a ese ingeniero en formación, dejar su cotidiano fuera de la clase de matemáticas, esto conlleva una falta de consideración de este conocimiento en la construcción de sus propias prácticas profesionales (GÓMEZ, 2015).

A pesar de que los programas educativos del mundo declaran que la matemática escolar es una herramienta para la vida cotidiana y para las profesiones, en general, se ignora cómo vive y cómo es usada en el cotidiano de las comunidades de conocimiento matemático (CORDERO, 2016a).

En el diagrama de la Figura 3 las flechas grises muestran la manera cíclica en que se cree debe funcionar el sistema educativo. El ingeniero agrónomo debe hacer del conocimiento matemático un instrumento para su vida cotidiana profesional. Por otro lado, el conocimiento cotidiano de esta comunidad de conocimiento matemático de ingenieros agrónomos (CCM (I.Agro)) debe ser una referencia que influya en la enseñanza y el aprendizaje de la matemática escolar (para los ingenieros), de tal manera que lo que se aprenda en la escuela tenga estrecha relación con la vida cotidiana de esa comunidad de ingenieros (GÓMEZ, 2015). 


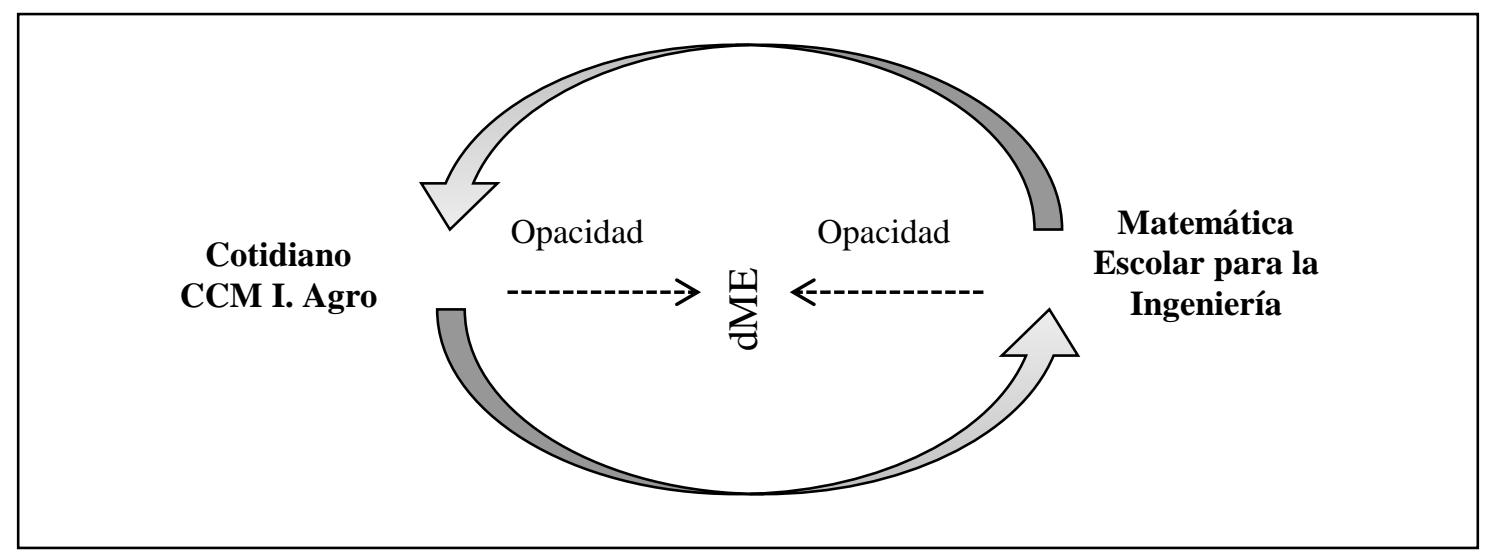

Figura 3 - Fenómeno de opacidad ocasionado por el dME Fuente: GÓMEZ (2015).

Sin embargo, la epistemología de la matemática escolar para la ingeniería y la epistemología de la matemática en el cotidiano de la CCM (I.Agro), se opacan mutuamente por causa del actual dME (GÓMEZ; CORDERO, 2013; GÓMEZ, 2015). Es decir, a pesar de que ambas epistemologías de conocimiento conviven en un mismo espacio, no se genera un diálogo recíproco y horizontal entre ellas para desarrollar la dualidad de la matemática escolar: como objeto de estudio racional y como herramienta funcional desde la CCM (I.Agro). El dME hace una centración al objeto soslayando el carácter funcional de la matemática (GÓMEZ, 2015).

Caracterizar el cotidiano propio de la CCM (I.Agro) está estrechamente relacionado con entender los funcionamientos y las formas del conocimiento matemático (CM) de la comunidad misma. Así, se analizan tres escenarios cercanos a esta comunidad: El aula. En particular las clases de Cálculo; El trabajo de campo. Durante actividades formativas del área y El disciplinar. Análisis de artículos y entrevista a un profesional.

Se distinguen al menos tres situaciones $(\mathrm{Si})$ donde se expresa la naturaleza del $\mathrm{CM}$ desde la CCM (I.Agro) (ver Cuadro 4):

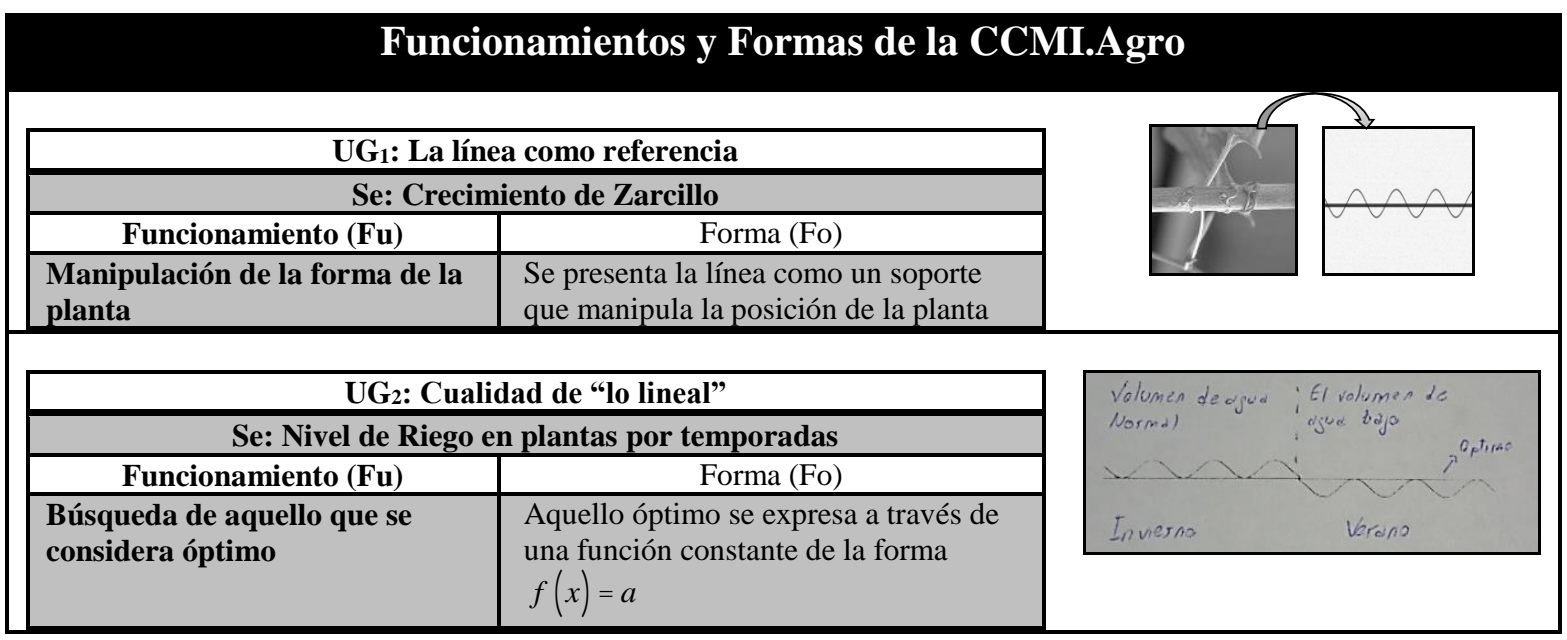




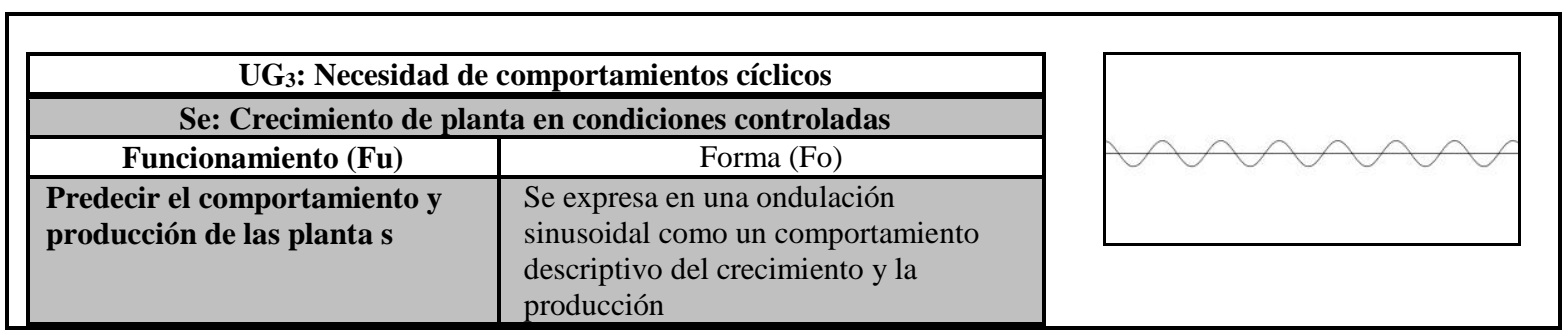

Cuadro 4 - Funcionamientos y Formas del CM de una CCMI.Agro

Fuente: GÓMEZ (2015).

Los funcionamientos y formas son las argumentaciones que expresan la naturaleza funcional del ingeniero agrónomo. La resignificación entre las tres situaciones presentadas se dará a través de la búsqueda de la optimización, es decir cuando lo lineal permite optimizar el sistema, en este caso de riego; y el ajuste a la estructura natural del sistema ya que es necesario analizar comportamientos cíclicos dada la naturaleza de los fenómenos que se estudian en la comunidad (ver Figura 4).

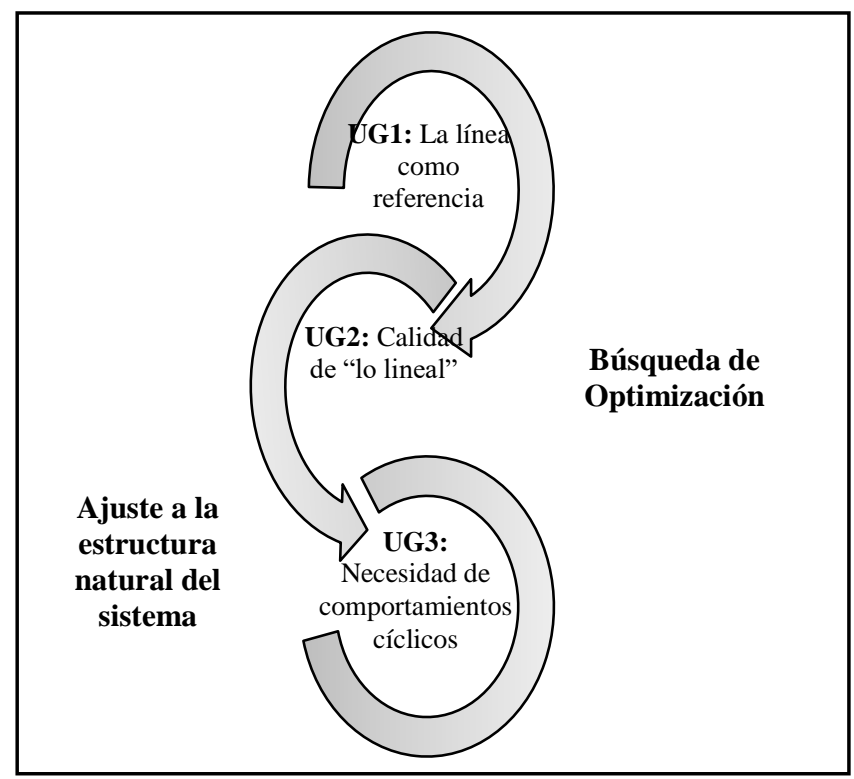

Figura 4 - Resignificación del CM desde una CCMI.Agro

Fuente: GÓMEZ (2015)

La práctica de modelación a partir de las Si de la CCM (I.Agro), expresa el carácter del CM como herramienta argumentativa que permite construir conocimiento desde su cotidiano profesional. Todo esto en contraparte de la matemática escolar, donde la matemática es centrada al objeto soslayando los usos y significados que emergen al seno de la CCM (I.Agro).

Así, la Teoría Socioepistemológica de la Matemática Educativa nos provee de elementos teóricos-metodológicos para rediseñar el $d M E$ (CANTORAL, 2013). Con las prácticas sociales se norma la construcción del conocimiento, las cuales se expresan en los usos y significados que emergen de los participantes para generar argumentaciones 
funcionales. Las argumentaciones son resignificaciones del CM en los diferentes escenarios transversales del conocimiento, por ejemplo la escuela, el trabajo y la ciudad (CORDERO, 2016a). Todo en conjunto, se podría considerar como una categoría de modelación que

permea los funcionamientos y las formas de los $U(\mathrm{CM})$, y hace visible el diálogo entre las pluralidades epistemológicas que el dME suele opacar (CORDERO, 2011; CORDERO et al., 2016).

\section{Conclusiones}

Del objeto a la funcionalidad matemática quiere decir integrar usos y significados al objeto matemático en una situación específica, en donde se debate entre la función y la forma de ese conocimiento, de acorde con lo que organizan los participantes. A este último se le llama resignificación. Así, la matemática funcional puede llevar a cabo múltiples realizaciones y hacer ajustes en su estructura para producir un patrón deseable. Esto significa que la funcionalidad es, por un lado, un medio que soporta el desarrollo del razonamiento y de la argumentación; y, por el otro, es una práctica que trasciende y se resignifica, que transforma al objeto en cuestión. De acuerdo a nuestras investigaciones, esa es la epistemología matemática que generan las comunidades de los ingenieros (SUÁREZ; CORDERO, 2010; CORDERO; MENA-LORCA; HUINCAHUE, 2017).

Así, la naturaleza de la situación Si que definirá la comunidad de conocimiento del ingeniero CC(Ii) corresponderá a una categoría de conocimiento matemático ( $(\mathrm{CM})$ ). Esta categoría pudiera ser la de modelación que hemos mencionado con antelación (CORDERO, 2011; CORDERO; MENA-LORCA; HUINCAHE, 2017).

Tal categoría tendrá que desarrollarse en el sistema educativo. Será el marco de referencia que ayude a resignificar el conocimiento matemático en los diferentes programas de formación de las ingenierías, en tanto matemática se refiere. Esta formulación creará una nueva base de entendimientos y construcciones donde la fuente de abstracción se encuentra en un ámbito de las prácticas.

Las categorías tendrán un carácter funcional del CM, de ahí la importancia del cotidiano. Esto es, una vez que se identifiquen las prácticas sociales, que dieron y dan cuenta del CM, requieren ser reinterpretadas para ser integradas al sistema didáctico, pues requieren de la intencionalidad para que se desarrollen en las condiciones del sistema. Para ello, se construye la situación donde la práctica se transforma en la argumentación, como el eje o núcleo para generar el CM que responda a la situación (CORDERO et al., 2015). 
Todo lo anterior expresa un cambio de paradigma el cual define un Programa Permanente (CORDERO, 2016a, CORDERO; MENA-LORCA; HUINCAHUE, 2017). Éste debe ser capaz de provocar la incidencia y la reproducción de la visión anteriormente planteada en el seno de los cuerpos académicos o grupos de investigación y en la red de investigadores y docentes que los mismos grupos van conformando (CORDERO; RODRÍGUEZ; SOLÍS, 2013).

El Programa tiene como tarea permanentemente vigilar los siguientes aspectos: la coordinación de acciones de la red de investigadores; la posibilidad de generar un mecanismo de vinculación tanto transdisciplinaria como multidisciplinaria; la realización de proyectos en colaboración con otras instituciones del país o del extranjero; desarrollar proyectos conjuntos en temas claves para el futuro del sistema educativo; y consolidar redes de investigadores de competencia internacional (CORDERO, 2016b).

\section{Referencias}

BARBOSA, J. C. Modelagem e Modelos Matemáticos na Educação Científica. Alexandria Revista de Educação em Ciência e Tecnologia, Brasil, v. 2, n. 2, p. 69-85, 2009.

BISSELL, C.; DILLON, C. Telling Tales: Models, Stories and Meanings. For the learning of Mathematics, Netherlands, v. 20, n. 3, p. 3-11, 2000.

BOYCE, W.; DIPRIMA, R. Ecuaciones diferenciales y problemas con valores en la frontera. México: Editorial Limusa, 1992.

BUENDÍA, G. La construcción social del conocimiento matemático escolar. Un estudio socioepistemológico sobre la periodicidad de las funciones. México: Editorial Díaz Santos, S.A, 2011. $138 \mathrm{p}$.

CAJAS, F. El conocimiento de ingeniería como conocimiento escolar. En: LESTÓN, P. (Ed.). Actas Latinoamericanas de Matemática Educativa 22, México: CLAME A.C., 2009. p. 77-84.

CANTORAL, R. Teoría Socioepistemológica de la Matemática Educativa. Estudios sobre construcción social del conocimiento. Barcelona: Gedisa, 2013. 370p.

CIVIL, M. Everyday Mathematics, mathematicians' mathematics, and school mathematics: can we bring them together? En: BRENER, M.; MOSCHKOVICH, J. (Ed.). Everyday and Academic Mathematics in the Classroom, Journal for Research in Mathematics Education. Reston, Virginia: National Council of Teachers of Mathematics [NCTM], 2002. p. 40-62.

CORDERO, F. El entendimiento de algunas categorías del conocimiento del cálculo y del análisis: el caso del comportamiento tendencial de las funciones Revista Latinoamericana de Investigación en Matemática Educativa, México, v. 1, n. 1, p. 56-74, 1998.

CORDERO, F. El uso de las gráficas en el discurso del cálculo escolar. Una visión socioepistemológica. En: CANTORAL, R. et al. (Ed.). Investigaciones sobre enseñanza y aprendizaje de las matemáticas: un reporte Iberoamericano, México: Díaz de Santos - CLAME. A. C., 2008. p. 265-286. 
CORDERO, F. La modelación y la graficación en la matemática escolar. En: RODRIGUEZSALAZAR, L. M.; QUINTERO, R.; HERNÁNDEZ, A. (Coord.). Razonamiento Matemático. Epistemología de la Imaginación. (Re)pensando el papel de la Epistemología en la Matemática Educativa. España, México: Gedisa, Cinvestav, 2011. p. 377-399.

CORDERO, F. Modelación, funcionalidad y multidisciplinariedad: el eslabón de la matemática y el cotidiano. En: ARRIETA, J.; DÍAZ, L. (Ed.). Investigaciones latinoamericanas de modelación de la matemática educativa. Barcelona: Gedisa, 2016a. p. 59-88.

CORDERO, F. La función social del docente de matemáticas: pluralidad, transversalidad y reciprocidad. En S. Estrella, M. et al., (Eds.), XX Actas de las Jornadas Nacionales de Educación Matemática. Valparaíso, Chile: SOCHIEM, IMA-PUCV. Recuperado de: http://ima.ucv .cl/congreso/xxjnem/. 2016b. p. 23-30.

CORDERO, F. La matemática y lo matemático. Transversalidad y modelación: un programa socioepistemológico. Documento en preparación. 2017.

CORDERO, F.; DUBINSKY, E.; FERRARI, M. Arreglos numéricos, transformaciones de figuras geométricas y comportamientos de las funciones como recursos del álgebra abstracta. México: Díaz de Santos, 2011, 98p.

CORDERO, F.; FLORES, R. El uso de las gráficas en el discurso matemático escolar. Un estudio socioepistemológico en el nivel básico a través de los libros de texto. Revista Latinoamericana de Investigación en Matemática Educativa, México, v. 10, n. 1, p. 7-38, 2007.

CORDERO, F. et al. El Discurso Matemático Escolar: la Adherencia, la Exclusión y la Opacidad. Barcelona, España: Gedisa, 2015. 148p.

CORDERO, F.; MENA-LORCA, J.; HUINCAHUE, J. A category of modeling: functional mathematics of other domains of knowledge and the learning of mathematics, 2017.

CORDERO, F.; MENA-LORCA, J.; MONTALTO M.E. Il ruolo della giustificazione funzionale in una situazione di risignificazione dell asintoto. l'insegnamento della Matematica, Italia, v. 33B, n. 4, p. 457-488, 2010.

CORDERO, F., RODRÍGUEZ, R. SOLÍS, M. Un programa de modelación para el Aprendizaje de la Matemática: la Escuela, el Trabajo y la Ciudad. Acta Latinoamericana de Matemática Educativa 26, México: CLAME A.C., 2013. p. 1267-1275.

CORDERO, F. et al. El comportamiento con tendencia, lo estable y las Ecuaciones Diferenciales Lineales. Una argumentación Gráfica. Barcelona, España: Gedisa, 2016. 143p.

CORDERO, F; SOLÍS, M. Las gráficas de las funciones como una argumentación del cálculo. $2^{\mathrm{a}}$. Edición. Cuadernos Didácticos, v. 2. México: Grupo Editorial Iberoamérica. 1997. 77p.

D’AMBROSIO, U. Etnomatemática e Educação. Reflexão e ação. Revista do Departamento de Educação, Santa Cruz do Sul, RS, v. 10, n. 1, p. 7-20, 2002.

EDWARDS, C. H.; PENNEY, D. Ecuaciones diferenciales elementales, con aplicaciones. México: Prentice Hall Hispanoamericana, 1993.

GÓMEZ, K. El fenómeno de opacidad y la socialización del conocimiento matemático. Lo matemático de la Ingeniería Agrónoma. 2015. 178f. Tesis (Doctorado en Ciencias Especialidad Matemática Educativa)-Departamento Matemática Educativa, CINVESTAV-IPN, México. 2015. 
GÓMEZ, K.; CORDERO, F. La institucionalidad, funcionalidad e historicidad. Elementos para el rediseño del discurso matemático escolar. En FLORES, R. (Ed.). Acta Latinoamericana de Matemática Educativa 26. México: CLAME A.C., 2013, p. 1323-1330.

HIRSH, C.; MCDUFFIE, A. Annual Perspectives in Mathematics Education: Mathematical Modeling and Modeling Mathematics. USA: National Council of Teachers of Mathematics, 2016. 280p.

KAISER, G.; SRIRAMAN, B. A global survey of international perspectives on modelling in mathematics education. Zentralblatt fur Didaktik der Mathematik, Berlín, Alemania, v. 38, n. 3, p. 302-310, 2006.

LARA, G.; CORDERO, F. Categorías de uso de las gráficas en ingeniería. En: CRESPO, C. (Ed.). Acta Latinoamericana de Matemática Educativa 20. México: CLAME A.C., 2007, p. 513-518.

MENDOZA-HIGUERA, E.J. Matemática funcional en una comunidad de conocimiento: el caso de las ecuaciones diferenciales lineales en la ingeniería, 2013. 76f. Tesis (Maestría en Ciencias Especialidad Matemática Educativa) - Departamento Matemática Educativa, Cinvestav-IPN, México. 2013.

MENDOZA-HIGUERA, E.J. La matemática funcional de una comunidad de conocimiento de ingenieros. El caso de la estabilidad en la electrónica. Documento Pre-doctoral (Doctorado en Ciencias Especialidad Matemática Educativa) - Departamento de Matemática Educativa,

CINVESTAV-IPN, 2017, 112p.

MENDOZA-HIGUERA, E.J.; CORDERO, F. El uso de las Ecuaciones Diferenciales y la ingeniería como Comunidad. En FLORES, R. (Ed.). Acta Latinoamericana de Matemática Educativa 25, México: CLAME A.C., 2012, p. 1023-1030.

MENDOZA-HIGUERA, E.J.; CORDERO, F. Matemática funcional en una comunidad de conocimiento. El caso de la estabilidad. III Coloquio de Doctorado, Departamento de Matemática Educativa, CINVESTA, México. 2015.

MORALES, A. et al. El rol del tiempo en un proceso de modelación utilizando videos de experimentos físicos. Revista Enseñanza de las Ciencias, España, v. 30, n. 3, p. 237-256, 2012.

MORALES, A.; CORDERO, F. La graficación-modelación y la Serie de Taylor. Una Socioepistemología del Cálculo. Revista Latinoamericana de Investigación en Matemática Educativa, México, v. 17, n. 3, p. 319-345, 2014.

NOSS, R.; HOYLES, C.;POZZI, S. Working Knowlegde: Mathematics in use. En: BESSOT, A.; RIDGWAY, J. (Ed.). Education for Mathematic in the Workplace. Holanda: Kluwer Academic Publischers, 2000. p.17-35.

PARRA, T.; CORDERO, F. El uso de las gráficas en la mecánica de fluidos. El caso de la derivada. En: CRESPO, C. (Ed.). Acta Latinoamericana de Matemática Educativa 20, México: CLAME A.C., 2007, p. 519-524.

SOARES, D.S. Um abordagem pedagógica baseada na análise de modelos para alunos de biología: qual o pelo do software? 2012. 341f. Tesis (Doctorado en Educación Matemática) - Instituto de Geociências e Ciências Torresias Exatas, Universidade Estadual Paulista, Brasil, 2012

SOLÍS, M. Estudio de la noción de variación en contextos físicos. El fenómeno de la propagación del calor. Premio Simón Bolívar 1997. México: Grupo Editorial Iberoamérica.1999. 150p. 
SOLÍS, M. Nociones de predicción y simulación en ecuaciones diferenciales a través del comportamiento tendencial de las funciones. En: CORDERO, F. (Ed.). Antologías 2. México: CLAME-RED DE CIMATES. 2002. p.113-136.

SOLÍS, M. Las gráficas de las funciones como una argumentación del cálculo. Caso de la predicción y la simulación en las ecuaciones diferenciales lineales de primer orden. 2012. 118f. Tesis (Doctorado en Ciencias Especialidad Matemática Educativa) - Departamento Matemática Educativa, CINVESTAV-IPN, México, 2012.

SUÁREZ, L.; CORDERO, F. Modelación-graficación, una categoría para la matemática escolar. Resultados de un estudio socioepistemológico. México. Revista Latinoamericana de Matemática Educativa, v. 14, n. 4-II, p. 319-333, 2010

TORRES, L. Usos del conocimiento matemático. La simultaneidad y la estabilidad en una comunidad de conocimiento de la ingeniería química en un escenario de trabajo. 2013. 137f. Tesis (Maestría en Ciencias Especialidad Matemática Educativa) - Departamento Matemática Educativa, CINVESTAV-IPN, México, 2013

TUYUB, I; CORDERO, F.; CANTORAL, R. Un estudio socioepistemológico en la práctica toxicológica. En: LESTÓN, P. (Ed.). Actas Latinoamericanas de Matemática Educativa 22, México: CLAME A.C., 2009, p. 1245-1254.

UNACH. Página web Facultad de ingeniería civil. México. Disponible en:<http://www.ingenieria .unach.mx/index.html>. Acceso en: 25 feb. 2018.

VÁZQUEZ, E. Funcionalidad de la estabilidad en la Biología. Un estudio socioepistemológico. 2011. 222f. Tesis (Maestría en Ciencias Especialidad Matemática Educativa) - Departamento Matemática Educativa, CINVESTAV-IPN, México, 2011.

ZALDÍVAR, D. et al. El espacio de trabajo matem’tico y la situación específica de la matemática funcional: un ejercicio de diálogo. Revista Latinoamericana de Investigación en Matemática Educativa, México, v, 17, n, 4-I, p. 191-210, 2014.

ZILL, D. Ecuaciones diferenciales, con aplicaciones de modelado. 6. ed. México: Internacional Thomson Editores, 1997. 